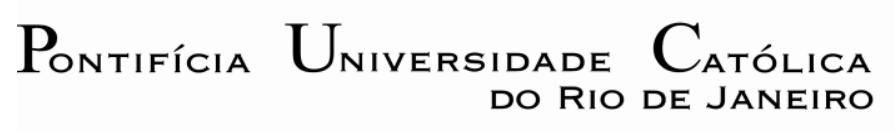

DO RIO DE JANEIRO

Eduardo Bevilaqua Pires

Análise Cross-Country do Impacto da Incorporação de Informação Macroeconômica na Previsão da Estrutura a Termo da Taxa de Juros

Dissertação de Mestrado

Dissertação apresentada como requisito parcial para obtenção do título de Mestre pelo Programa de PósGraduação em Ciências Atuariais do Instituto de Gestão de Riscos Financeiros e Atuariais da PUCRio.

Orientador: Prof. Luciano Vereda Oliveira

Rio de Janeiro, março de 2009 


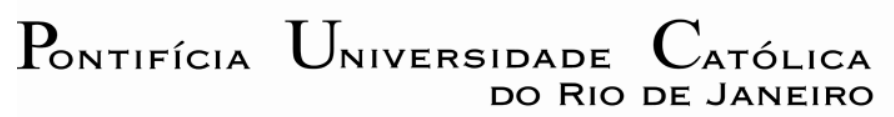

DO RIO DE JANEIRO

Eduardo Bevilaqua Pires

\section{Análise Cross-Country do Impacto da Incorporação de Informação Macroeconômica na Previsão da Estrutura a Termo da Taxa de Juros}

Dissertação apresentada como requisito parcial para obtenção do título de Mestre pelo Programa de PósGraduação em Ciências Atuariais do Instituto de Gestão de Riscos Financeiros e Atuariais da PUC-Rio.

Prof. Luciano Vereda Oliveira Orientador Presidente Instituto de Gestão de Riscos Financeiros e Atuarias - PUC-Rio

Prof. Cristiano A. C. Fernandes Departamento de Engenharia Elétrica - PUC-Rio

Prof. Helio C. Vieira Lopes

Departamento de Matemática - PUC-Rio

Prof. Nizar Messari

Coordenador Setorial do Centro de Ciências Sociais - PUC Rio 
Todos os direitos reservados. É proibida a reprodução total ou parcial do trabalho sem autorização do autor, do orientador e da universidade.

\section{Eduardo Bevilaqua Pires}

Graduado como Bacharel em Ciências Econômicas pela Pontifícia Universidade Católica do Rio de Janeiro (PUC-Rio) em 2006.

Ficha Catalográfica

Pires, Eduardo Bevilaqua

Análise cross-country do impacto da incorporação de informação macroeconômica na previsão da estrutura a termo da taxa de juros / Eduardo Bevilaqua Pires ; orientador: Luciano Vereda Oliveira. - 2009.

$50 \mathrm{f.} ; 30 \mathrm{~cm}$

Dissertação (Mestrado em Gestão de Riscos Financeiros e Atuariais) - Pontifícia Universidade Católica do Rio de Janeiro, Rio de Janeiro, 2009.

Inclui bibliografia

1. Gestão de Riscos Financeiros e Atuariais - Teses. 2. Estrutura a termo de taxa de juros. 3. Modelos autoregressivos. 4. Previsão. 5. Títulos. 6. Maturidades. 7. Variáveis macroeconômicas. I. Oliveira, Luciano Vereda. II. Pontifícia Universidade Católica do Rio de Janeiro. Instituto de Gestão de Riscos Financeiros e Atuariais. III. Título. 


\section{Agradecimentos}

Agradeço à Capes e ao IAPUC pela oportunidade de cursar o mestrado, pelo auxílio financeiro e incentivo à pesquisa.

Ao professor Luiz Roberto Cunha, diretor do IAPUC, por todas as oportunidades que me deu e, principalmente, a confiança e amizade em mim depositados.

Ao meu orientador, Luciano Vereda, por ter sido sempre compreensivo, me motivado, e muitas vezes ter aberto mão de parte de seu fim de semana para me receber.

Ao Professor Cristiano Fernandes, coordenador acadêmico do IAPUC, pelos cursos ministrados, e a paciência e compreensão que teve comigo.

Aos meus pais e minha irmã, que não só me apoiaram incondicionalmente em todos os momentos, me aturaram durante os dois anos de matérias cursadas e, principalmente, viabilizaram tantas idas minhas ao Rio de Janeiro, para que pudesse concluir minha tese de maneira satisfatória.

Aos meus amigos queridos, Luciano e Alessandra. Vocês valem ouro.

Às Anas, Luiza e Tereza, por todo o incentivo, puxões de orelha, revisões e comentários no texto e auditoria nos resultados. Foi bem mais fácil com a companhia e carinho de vocês.

À Luciene, que esteve presente em todas as etapas do mestrado, sempre nos ajudando e trazendo alegria para nossa rotina de estudos!

Ao Unibanco, na pessoa de Felipe Valente, que me liberou para sair mais cedo nas 6as feiras de idas ao RJ, permitiu que eu desse um gás nas últimas três semanas, e por jamais ter me repreendido quando cheguei atrasado por ter dormido de madrugada, após a maratona de tese.

Por fim, aos meus amigos queridos, por terem sido sempre tão presentes, compreensivos e prestativos: Olavo Vaz, Caroline Martins, Guilherme Giorelli, Bernardo Medeiros, Conrado Ferraz, Felipe Novaes, Eduardo Jorge, Guilherme Cardoso, Rodrigo Eboli, Tiago Pitella, Clara Iglesias, Pedro Valente, Cecília Vieira de Melo, Breno Vaz, Alexandre Azevedo, Bruno Guimarães, Diego Tavares, Carlos Gustavo, Hermínia Calmon, Diego Tavares, Maria Clara Kurban, Marcela Tapia, Guto, Ana Ghislane, Juliana Correa, Diana Litewski, Fernanda Cunha, Rachel Kovacs, Veridiana Caldas, Clara Roorda, Eduardo Karpat, Tatiana Soares, Paula Braga, Renato Sigoli, Guilherme Bockmann, Mayra Toledo, Bruno Carraro, Rafael Tonetti, Enrico Chiavegato, Claudio Tarrasco, Renato Gradia, Reimy Okazaki e Ana Paula Amaral. 


\section{Resumo}

Pires, Eduardo Bevilaqua; Oliveira, Luciano Vereda (Orientador). Análise Cross-Country do Impacto da Incorporação de Informação Macroeconômica na Previsão da Estrutura a Termo da Taxa de Juros . Rio de Janeiro, 2009. 50p. Dissertação de Mestrado - Departamento de Ciências Atuariais, Pontifícia Universidade Católica do Rio de Janeiro.

No Brasil e no mundo, grande parte das carteiras de investimento das seguradoras e entidades de previdência complementar é composta por títulos emitidos por governos. Sendo assim, os retornos destes papéis respondem por parte relevante da rentabilidade destas carteiras. Este trabalho tem como finalidade a análise do impacto da incorporação de informações econômicas (taxa de juros, hiato do produto e taxa de inflação) na previsão da Estrutura a Termo de Taxa de Juros de quatro países: EUA, Reino Unido, Brasil e Chile. Diferentes modelos de vetores auto-regressivos (VAR) são testados e comparados com outros modelos, como passeio aleatório e modelos auto-regressivos (AR), em termos de performance de previsão out-of-sample

\section{Palavras-chave}

Estrutura a Termo de Taxa de Juros; Modelos Auto-regressivos; Previsão; Títulos; Maturidades; Variáveis Macroeconômicas. 


\section{Abstract}

Pires, Eduardo Bevilaqua; Oliveira, Luciano Vereda (Advisor). CrossCountry Analysis of the Effects of Introducing Macroeconomic Information to Yield Curve Forecasts. Rio de Janeiro, 2009. 50p. MSc Dissertation - Departamento de Ciências Atuariais, Pontifícia Universidade Católica do Rio de Janeiro.

In Brazil and the world, much of the investment portfolios of insurers and complementary pension funds consists of securities issued by governments. Thus, the returns of these papers account for the relevant part of the profitability of these portfolios. This work aims at analyzing the impact of the incorporation of economic data (interest rate, output gap and inflation rate) in the forecast of the Term Structure of Interest Rates in four countries: USA, UK, Brazil and Chile. Different models of vector autoregression (VAR) are tested and compared with other models such as random walk and autoregressive models (AR), in terms of performance of out-of-sample forecasts."

\section{Keywords}

Term Structure of Interest Rates; Auto-regressive Models; Forecast; Bonds; Maturities; Macroeconomic Variables. 


\section{Sumário}

1. Introdução 10

2. Metodologia e revisão de literatura 13

2.1. Vetores Auto-regressivos 13

2.2. O modelo de Evans e Marshall 15

2.3. Metodologia aplicada 18

2.3.1. Primeiro modelo - VAR 121

2.3.2. Segundo modelo - VAR 2 22

2.3.3. Terceiro modelo - VAR 3 23

2.3.4. Quarto modelo - VAR 4 24

2.3.5. Quinto modelo - VAR 5 25

2.3.6. Sexto modelo - Eq. c/ Variáveis Macro Previstas 26

2.3.7. Sétimo modelo - Eq. c/ Variáveis Macro Observadas 27

$\begin{array}{ll}\text { 2.3.8. Modelo auto-regressivo } & 27\end{array}$

3. Descrição de dados 29

4. Resultados 38

4.1. Erro quadrático médio 38

4.2. Erro absoluto 43

5. Conclusão 47

6. Referências bibliográficas 49 


\section{Lista de figuras}

Figura 1. Estrutura a Termo de Taxa de Juros dos EUA 30

Figura 2. Estrutura a Termo de Taxa de Juros da Inglaterra 31

Figura 3. Estrutura a Termo de Taxa de Juros do Chile 31

Figura 4. Estrutura a Termo de Taxa de Juros do Brasil 32

Figura 5. Variáveis macroeconômicas dos EUA: taxa de juros, inflação e hiato do produto 33

Figura 6. Variáveis macroeconômicas da Inglaterra: taxa de juros, inflação e hiato do produto 34

Figura 7. Variáveis macroeconômicas do Chile: taxa de juros, inflação e hiato do produto 35

Figura 8. Variáveis macroeconômicas do Brasil: taxa de juros, inflação e hiato do produto 36 


\section{Lista de tabelas}

Tabela 1. Estatística descritiva das séries de retorno dos títulos de todos os $\begin{array}{ll}\text { países } & 30\end{array}$

Tabela 2. Matriz de correlações das séries de retorno dos títulos de todos os países 30

Tabela 3. Erro quadrático médio para as previsões um passo à frente $\quad 40$

Tabela 4. Erro quadrático médio das previsões seis passos à frente 41

Tabela 5. Erro quadrático médio das previsões doze passos à frente 43

Tabela 6. Erro absoluto das previsões um passo à frente 44

Tabela 7. Erro absoluto das previsões seis passos à frente 45

Tabela 8. Erro absoluto das previsões doze passos à frente 46 